\title{
AVALIAÇÕES DE PROGRAMAS GOVERNAMENTAIS REALIZADAS PELO TRIBUNAL DE CONTAS DA UNIÃO
}

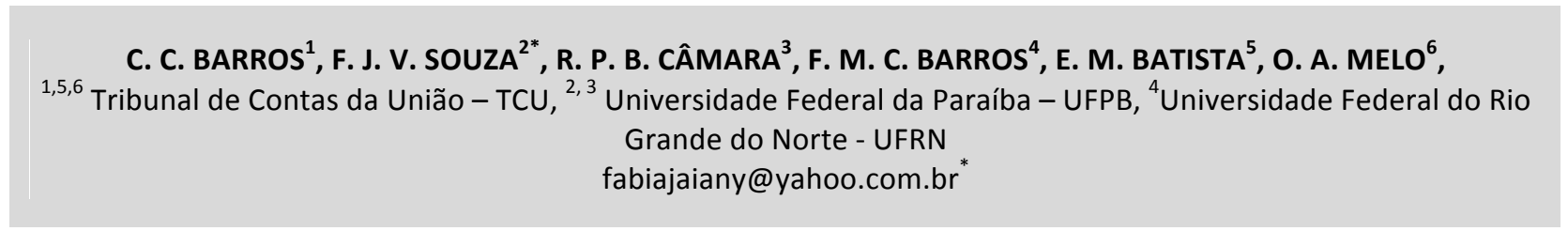 \\ Submetido 25/01/2018 - Aceito 27/06/2018 \\ DOI: $10.15628 /$ holos.2018.5854
}

\section{RESUMO}

Esta pesquisa objetivou identificar a atuação do TCU na avaliação de programas governamentais em funções do governo no período 2000-2012. Para tanto, a metodologia adotada consistiu de pesquisas descritivas, documental e qualitativa. Os resultados revelaram que as 73 avaliações de programas governamentais analisadas foram executadas por 490 auditores, utilizando 6 estratégias metodológicas, 14 técnicas de diagnóstico, 4 tipos de coleta de dados e 6 formas de analisá-los, consumindo em média 117,83 HDFs (Homem Dia Fiscalização). Conclui-se que as avaliações de programas do TCU estão em sintonia com a tendência mundial contribuindo com a APFB tendo ofertado 1.373 sugestões, cujo impacto nos programas públicos foi advindo da implementação de 435 destas recomendações. Contudo, a despeito da avaliação positiva quanto aos benefícios gerados pela atuação do TCU, a pesquisa identificou que a demanda por este tipo de auditoria, por parte dos congressistas, não está alinhada ao comportamento mundial (NAO, GAO, Corte de Contas da Alemanha e da França).

PALAVRAS-CHAVE: Avaliação, Programas Públicos, TCU.

\section{REVIEWS OF GOVERNMENT PROGRAMS CONDUCTED BY TRIBUNAL DE CONTAS DA UNIÃO}

\begin{abstract}
This research aimed to identify the performance of the TCU in the evaluation of government programs in government functions during the period. For this, the methodology adopted consisted of descriptive, documentary and qualitative research. The results revealed that the 73 evaluations of government programs analyzed were performed by 490 auditors, using 6 methodological strategies, 14 diagnostic techniques, 4 types of data collection and 6 ways of analyzing them, consuming an average of 117.83 HDFs
\end{abstract}

Day Inspection). It is concluded that TCU program evaluations are in line with the worldwide trend contributing to the APFB and offered 1,373 suggestions, whose impact on the public programs came from the implementation of 435 of these recommendations. However, despite the positive evaluation of the benefits generated by the TCU's performance, the research identified that the demand for this type of audit by congressmen is not in line with world behavior (NAO, $\mathrm{GAO}$, Court of Accounts of Germany and from France).

KEYWORDS: Evaluation, Government Programs, TCU. 


\section{INTRODUÇÃO}

As mudanças econômicas, sociais e políticas ocorridas na atualidade têm como um de seus fatores motivadores a ineficiência da gestão pública. Para Souza e Barros (2013), essas mudanças têm despertado a visão crítica da sociedade levando-a a uma participação mais efetiva quanto aos rumos das políticas públicas. Além de buscar dos gestores governamentais, uma crescente melhoria dos serviços ofertados a população, bem como uma atuação transparente da gestão pública.

Moore (1995) explica que os cidadãos estão perdendo a confiança em seus governos, e ampliando sua confiança no setor privado, uma vez que as empresas privadas têm promovido o desenvolvimento econômico das localidades onde atuam (Matias-Pereira, 2010). Nessa conjuntura, a relação do Estado com o setor privado foi significativamente alterada a partir da década de 1990, por influência do movimento da New Public Management (NPM). A Nova Gestão Pública incorpora um modelo de gestão voltado para a eficiência, eficácia e efetividade do aparelho do Estado. A NPM caracteriza-se por uma visão balizada sob a incorporação de instrumentos gerenciais do setor privado ao setor público (Rezende, Cunha \& Cardoso, 2010).

A nova tendência de gestão pública exige do controle governamental uma adaptação a esse conjunto de mudanças. As dimensões de eficiência, eficácia e efetividade foram inseridas no âmbito do controle governamental, quando a Constituição Federal incluiu a responsabilidade de fiscalizações ao Tribunal de Contas da União (TCU). Esse, em atendimento, criou a auditoria operacional (ANOp).

A auditoria operacional tem como uma de suas modalidades, a avaliação de programas, que se constitui na coleta e análise sistemática de informações sobre características, processos e impactos dos programas, atividades ou organizações (Brasil, 2012). Para Barzelay (1992), a Nova Gestão Pública oferece um arcabouço teórico para ampliar a competência legal das instituições responsáveis por auditorias governamentais, de forma, a incluir a avaliação de programas como melhoria do desempenho dos programas governamentais.

Alinhando-se a tendência mundial, o TCU criou sua unidade especializada, denominada Secretaria de Fiscalização e Avaliação de Programas de Governo (Seprog) no ano 2000, seguindo os passos de organizações de natureza análoga, quais sejam: no Reino Unido, a Comissão de Auditoria para a Inglaterra e País de Gales (Audit Comission for England and Wales), o National Audit Office (NAO), O Office of Standards in Education (Scott, 1995; Hood, 1996). Rist (1989), ao comparar os pontos fortes e fracos da auditoria como meio de monitoramento da prestação de contas, concluiu que o General Accounting Office - GAO tem priorizado avaliação de programas, em detrimento a auditoria tradicional.

Diante desse contexto, emerge a seguinte questão: Qual é a atuação do TCU na avaliação de programas governamentais em funções do governo no período 2000-2012? É, portanto, objetivo desta pesquisa identificar a atuação do TCU na avaliação de programas governamentais em funções do governo no período 2000-2012.

A relevância da pesquisa consiste em identificar o quantitativo dos resultados da atuação do TCU na avaliação de programas governamentais, com a perspectiva de poder aperfeiçoar as 
políticas públicas, apresentando o detalhamento dessas avaliações em funções básicas e estratégicas do governo.

Em sua dimensão social, a pesquisa contribui para que a população possa ter acesso a informações que a auxilie na fiscalização da aplicação dos recursos públicos. Vale ressaltar, o fato dos resultados desse estudo proporcionarem subsídios que possam ser utilizados na discussão e formulação de políticas públicas, assim como, em abordagens teóricas referentes à avaliação de ações governamentais implementadas pelo governo brasileiro.

\section{AVALIAÇÃO DE PROGRAMAS GOVERNAMENTAIS}

A avaliação de programas é um tema recente no Brasil. A sua institucionalização ocorreu por meio da Constituição de 1988. Após o surgimento da Nova Gestão Pública, o governo federal lançou o Plano Diretor da Reforma do Aparelho do Estado no ano de 1995. Esse plano criou, entre suas atribuições, o Sistema de Informações Gerenciais (SIG), tendo o objetivo de acompanhar os 42 programas prioritários integrantes do plano de metas "Brasil em Ação". O referido modelo de gestão passou a exigir um controle governamental baseado no exame dos resultados, com análise das dimensões de eficiência, eficácia e efetividade. A partir desse momento, surgiu a auditoria operacional e, por consequência dela, a auditoria de desempenho e avaliação de programas.

A auditoria de desempenho tem sido utilizada para distinguir a auditoria tradicional da avaliação de programas. Ainda não há um consenso mundial referente ao conceito de auditoria de desempenho (Barzelay, 1997; Vieira \& Gomes, 2002). Alguns órgãos centrais de auditoria tendem a destacar as semelhanças entre auditoria tradicional e auditoria de desempenho, provavelmente, para manter ou expandir a auditoria tradicional (SEDGWICK, 1993). Acrescentase a isso, o fato de que especialistas em avaliação caracterizam a auditoria de desempenho como uma forma de avaliação (Rist, 1989).

Pollitt et al. (1999) desenvolveu um modelo no qual a auditoria de desempenho pode estar relacionada com o atendimento dos seguintes critérios: economia, eficiência, efetividade, boa prática de gestão, boa governança, qualidade de serviço e atendimento de metas. Os autores explicam que o critério da boa prática de gestão constitui a grande parte dos trabalhos de auditoria. Segundo os mesmos autores, a boa prática de gestão consiste em testes que verifiquem se o auditado possui um bom sistema de contabilização, com procedimentos de determinação de metas, se existe uma boa relação com os clientes e se utilizam o benchmarking para elaborar modelos ou padrões de bom gerenciamento.

Barzelay (1997) discorre que os critérios de auditoria podem ser vistos como subcategorias ou tipos de auditoria de desempenho, tendo identificado os seguintes: eficiência, efetividade, capacidade de gerenciamento do desempenho, informações de desempenho, revisões da melhor prática de gestão, avaliação de risco e revisões gerais de gestão. O modelo foi testado em 13 países da Organização de Cooperação para o Desenvolvimento Econômico (OCDE).

A avaliação de programas é definida no sentido de intervenção social e de forma sistemática por Aguilar \& Ander-Egg (1994, p. 23) como sendo "procedimentos que, apoiados no 
uso de método científico, servem para identificar, obter e proporcionar a informação pertinente e julgar o mérito e o valor de algo de maneira justificável".

Para Weiss (1998), a avaliação é uma análise sistemática de processo e/ou dos resultados de um programa ou política, tendo o objetivo de contribuir para o seu aperfeiçoamento. Essa definição pode ser decomposta em cinco elementos: o primeiro diz respeito à análise sistemática, em que se enfatiza a natureza metodológica do trabalho; o segundo e o terceiro referem-se ao foco da investigação, ou seja, o processo de execução ou os resultados do programa e/ou política; o quarto trata dos critérios, que são os padrões de comparação; e o quinto se relaciona ao propósito da avaliação.

Conforme Calmon (1999), a avaliação de programas é um dos mais importantes instrumentos de feedback para os programas governamentais, uma vez que contribuem para aprendizagem organizacional, fornecendo dados que podem aperfeiçoar a gestão das políticas públicas. Apesar da relevância dessa temática para o contexto governamental, são raros os trabalhos dedicados à avaliação de programas utilizando como fonte de pesquisa as avaliações realizadas pelo TCU.

Dentre eles, destaca-se a tese de Hedler (2007), que produziu uma meta-avaliação das avaliações de programas realizadas pelo Tribunal de Contas da União. Ao final da pesquisa, o autor concluiu que as avaliações dos programas estão associadas a variáveis como contexto político, características dos programas sociais, enfoques, métodos e técnicas de auditoria.

Batista et al. (2014), realizou uma pesquisa semelhante a esta, entretanto, restringiu-se as avaliações de programas realizadas na área da saúde. Os resultados da pesquisa revelaram a realização de 17 avaliações que contaram com 121 auditores, sendo 65,29\% do gênero masculino e o emprego de 17 tipos de técnicas de diagnóstico, predominando a entrevista e a pesquisa postal/eletrônica. Ao final da pesquisa, concluiu que as avaliações de programas realizadas pelo TCU foram convertidas em subsídios para o aperfeiçoamento de estratégias futuras para a gestão da área da saúde.

A avaliação de programas é um mecanismo que possibilita analisar se a execução das políticas públicas está sendo eficaz ao que se propõe. Sabendo-se da relevância desse tipo de investigação, verifica-se a necessidade de estudos que analisem a avaliação de programas governamentais realizados nas diversas funções do governo, com a perspectiva que a quantificação de seus resultados possibilite a sociedade mapear a atuação do TCU na avaliação dos programas de áreas básicas e estratégicas do governo.

\section{PROCEDIMENTOS METODOLÓGICOS}

\subsection{Tipologias da Pesquisa}

Com base em Raupp e Beuren (2012), o delineamento da pesquisa pode ser classificado em três categorias: quanto aos objetivos, procedimentos e abordagem do problema. 
Quanto aos objetivos, esta pesquisa caracteriza-se como sendo de natureza descritiva, tendo em vista que busca descrever a atuação do TCU na avaliação de programas governamentais em funções do governo no período 2000-2012.

Com relação aos procedimentos, esse estudo pode ser classificado como uma pesquisa documental, uma vez que para a elaboração do estudo foram analisados os relatórios emitidos pelo TCU que versam sobre as avaliações dos programas governamentais.

Quanto à abordagem do problema, a pesquisa é qualitativa, pois não foram utilizados métodos estatísticos para a execução da análise do objeto do estudo.

\subsection{Amostra da Pesquisa}

Para compreender a amostra de pesquisa do estudo, se faz necessário apresentar as fases que corresponderam a implantação das auditorias operacionais nas avaliações de programas realizadas pelo TCU ao longo dos anos.

Após receber da Constituição Brasileira (Brasil, 1988), as atribuiç̧̃̃es de auditoria operacional, o TCU intensificou os treinamentos sobre essa temática, capacitando 600 servidores, entre técnicos, assessores, dirigentes e representantes dos Tribunais de Contas Estaduais e Municipais. Além de elaborar os primeiros normativos e executar 44 auditorias com esse escopo. Esse período correspondeu a 1a fase.

O TCU capacitou 26 auditores em Washington (EUA), objetivando se adequar ao cenário mundial, o qual denominou Projeto de Capacitação em Avaliação de Programas Públicos, desenvolvido com o apoio da Fundação Getúlio Vargas (FGV), em parceria com a Virginia Polytechinic Institute and State University e a National Academy of Public Administration (NAPA). Após o treinamento (1997), foram realizadas 9 avaliações-piloto, denominada de 2a fase.

Em abril/1998, o TCU firmou o Projeto de Aperfeiçoamento do Controle Externo com Foco na Redução das Desigualdades (CERDS), que se materializou por meio do Acordo de Cooperação Técnica entre o Governo do Brasil e o Governo do Reino Unido da Grã-Bretanha e Irlanda do Norte, denominado Projeto TCU/Reino Unido. Essa cooperação tinha como foco para a implantação do Projeto de Desenvolvimento de Técnicas de Auditoria Operacional, nas áreas de Saúde, Educação, Meio Ambiente, Assistência Social e Agricultura, com vigência de abril/1998 a dezembro/2002 (Júnior Benjamin, 2005). O Projeto TCU/Reino Unido contabilizou 24 auditoriaspiloto (3a fase).

Como universo da pesquisa, tem-se 154 avaliações de programas, sendo 44 (1a fase) + 9 (2 a fase) + 24 (3a fase) Projeto/Reino Unido (1998-2002) + 73 realizadas pela Seprog (2002-2012) e quatro realizadas em 2013, após a extinção da Seprog. Para a amostra da pesquisa, foram selecionadas as 73 avaliações de programas governamentais realizadas pela Seprog (2002-2012), uma vez que as outras 77 ( $1 \underline{a}+2 \underline{a}+3 \underline{a}$ fases) são consideradas como estudo/aprendizagem, e as 4 avaliações, feitas em 2013, não foram executadas pela secretaria especializada. 


\subsection{Coleta de dados}

A coleta de dados foi realizada na página eletrônica do TCU, onde foi possível ter acesso aos relatórios das avaliações governamentais para o período analisado. Após obter os relatórios, buscou-se identificar as técnicas de diagnóstico utilizadas. Com auxílio da ferramenta de busca localizar, foram identificados os nomes (SWOT, Árvore de Problemas, Coso, Ishikawa, DVR, Mapa Cognitivo, Matriz de Riscos, Benchmarking, Mapa de Produtos, Indicadores de Desempenho (ID), Mapa de Processo, Stakeholder, RECl e Marco Lógico) das técnicas de auditoria utilizadas nas avaliações governamentais pesquisadas. Realizou-se, também, uma leitura do capítulo metodologia, com o objetivo de identificar as estratégias metodológicas empregadas e a forma de coletar e analisar os dados.

Verificou-se, que a Seprog realiza o Painel de Referência, que é dividido em dois momentos. O primeiro, ao final da fase de planejamento, para validar os papeis de trabalho elaborados e a matriz de planejamento. O segundo, ao final da fase de execução, com o intuito de validar os achados. As contracapas e a primeira folha dos Relatórios de Avaliação foram investigadas com o intuito de identificar as equipes de auditoria, organizando-os por gênero, bem como indicando se houve a participação de membros externos e de onde vieram (Tribunal de Contas dos Estados (TCE), Controladoria-Geral da União (CGU), Advocacia Geral do Estado de Minas Gerais (AGE/MG) etc.). Os Acórdãos foram lidos e analisados identificando a quantidade de recomendações, sendo expurgado as determinações internas ao TCU.

O TCU desenvolveu a variável HDF (Homem Dia Fiscalização), para medir a relação entre os dias trabalhados e os auditores utilizados nas auditorias. As informações do HDF foram coletadas do Sistema Sinergia. Esse sistema foi desenvolvido pelo TCU para disponibilizar suas informações nos sistemas corporativos.

O art. 71 da Constituição Federal expressa que o controle externo, a cargo do Congresso Nacional (CN), será exercido com o auxílio do TCU. Portanto, é importante conhecer as demandas oriundas do CN para as avaliações de programa. Os dados para esta pesquisa foram extraídos das publicações TCU em Números, edições 2000-2009, e do Sinergia (2010-2012).

As informações coletadas e acompanhadas de procedimentos analíticos que acompanharam o transcorrer da pesquisa desde o momento em que se verificou a pertinência da questão selecionada ao objeto da pesquisa até as considerações finais.

\section{RESULTADOS DA PESQUISA}

\subsection{Programas Governamentais avaliados pelo TCU}

O TCU realizou 73 avaliações de programas governamentais no período de 2002 a 2012. Essas avaliações estão agrupadas em 21 áreas, as quais são definidas pelo próprio TCU. Foram identificados 314 Programas no PPA 2012-2015. Desse grupo foram analisados 23,24\% programas32. A Tabela 1 apresenta a quantidade de avaliações de programas realizada por área de atuação do Governo Federal. 
Tabela 1: Quantidade de avaliações de programas realizadas por área de atuação do governo no período 2002-2012

\begin{tabular}{c|c|c}
\hline Área das auditorias & Quantidade & Percentual (\%) \\
\hline Saúde & 17 & 23,29 \\
Assistência Social & 10 & 13,70 \\
Cidadania & 7 & 9,59 \\
Agricultura & 4 & 5,48 \\
Transporte & 4 & 5,48 \\
Educação & 3 & 4,11 \\
Esportes & 3 & 4,11 \\
Gestão Ambiental & 3 & 4,11 \\
Habitação & 3 & 4,11 \\
Industria e Comércio & 3 & 4,11 \\
Ciência e Tecnologia & 2 & 2,74 \\
Relações Exteriores & 2 & 2,74 \\
Saneamento e & & \\
Urbanismo & 2 & 2,74 \\
Segurança & 2 & 2,74 \\
Trabalho & 2 & 2,74 \\
Comunicações & 1 & 1,37 \\
Direitos Humanos & 1 & 1,37 \\
Energia Elétrica & 1 & 1,37 \\
Organização Agrária & 1 & 1,37 \\
Previdência Social & 1 & 1,37 \\
Administração Pública & 1 & 1,37 \\
Total & 73 & 100,00 \\
\hline
\end{tabular}

Fonte: dados da pesquisa (2013)

Com base na análise realizada da Tabela 1, verifica-se que o TCU priorizou a área de saúde como aquela com o maior número de avaliações de programas governamentais realizadas no período de análise. Essa área obteve de 17 avaliações, a qual foi seguida pelas áreas de Assistência Social e Cidadania, que apresentaram, respectivamente, 10 e 7 avaliações. Esses resultados podem ser justificados pelo fato dessas áreas serem consideradas pela Seprog como áreas básicas e estratégicas do Governo Federal. Tal situação favorece que uma quantidade maior de avaliações seja realizada para programas governamentais pertencentes a essas áreas.

Quando é observada a média de avaliações por áreas de atuação do Governo Federal, identifica-se que a média correspondeu a 3,47 avaliações. Acrescenta-se a essas informações, que as avaliações dos programas governamentais das áreas de Educação, Esportes, Gestão Ambiental, Habitação, Saneamento e Urbanismo, Indústria, Comércio e Serviços, que registraram 3 avaliações, Ciência e Tecnologia, Relações Exteriores, Saneamento, Segurança e Trabalho, com 2 avaliações, e Administração Pública, Ciência e Tecnologia, Comunicações, Direitos Humanos, Energia Elétrica, Previdência Social, com 1 avaliação estiveram abaixo dessa média.

Esses resultados permitem inferir que a Seprog optou por centrar seus esforços de atuação em avaliações de áreas governamentais que são essenciais para a sociedade, assim 
como, aquelas que concentram uma parcela alta do orçamento federal. Entretanto, essa inferência não quer dizer que as três áreas com maior número de avaliações são mais relevantes que as demais. Porém, pela natureza de seu objeto, motivaram a Seprog por selecioná-las como aquelas que deveriam ser priorizadas por seu retorno mais imediato para a sociedade.

Quando é observado o número de avaliações realizadas pelo TCU no período de análise, identifica-se que a quantidade de auditorias correspondeu ao intervalo de 2 a 11 avaliações. Foi possível verificar que a média de avaliações por ano pesquisado foi de 6,63. Além disso, constatou-se uma variação entre quantidades mínima e máxima de 9 avaliações $(11-2=9$ ). Esses resultados, permitem inferir que ocorreu um crescimento de 35,61\% em relação a média.

Verificou-se, também, que a produtividade de avaliações dos programas governamentais ficou acima da média nos exercícios de 2004, 2006 e, entre 2009 a 2012. Acrescenta a essa análise, que somente as áreas de Saúde (17), Assistência Social (10) e Cidadania (7) foram avaliadas acima da média. Essa constatação permite inferir um índice baixo de avaliação de programas governamentais por áreas específicas, o que não contribui para a expansão deste tipo de auditoria.

\subsection{Técnicas de diagnóstico dos programas governamentais}

O estudo permitiu observar que das 73 avaliações de programas governamentais realizadas no período de 2002 a 2012 foram utilizadas 14 técnicas de diagnóstico. A Tabela 2 a quantidade de técnicas utilizadas nas avaliações pesquisadas.

Tabela 2: Técnicas de diagnóstico utilizadas nas avaliações de programas governamentais

\begin{tabular}{c|c|c}
\hline Técnicas de & $\begin{array}{c}\text { Quantidade de } \\
\text { avaliações }\end{array}$ & Percentual (\%) \\
\hline Diagnóstico & 14 & 19,17 \\
Análise de Stakeholder & 4 & 5,48 \\
Árvore de Problemas & 5 & 6,84 \\
Benchmarking & 11 & 15,06 \\
Coso & 4 & 5,48 \\
Diagrama de Ishikawa & 13 & 17,80 \\
DVR & 59 & 80,82 \\
Indicador de & 1 & 1,36 \\
Desempenho (ID) & 18 & 24,66 \\
Mapa Cognitivo & 6 & 8,21 \\
Mapa de Processos & 3 & 4,10 \\
Mapa de Produtos e & 2 & 2,73 \\
Impactos & 1 & 1,36 \\
Marco Lógico & 20 & 27,39 \\
Matriz de Riscos & & \\
RECl & & \\
SWOT & & \\
\hline
\end{tabular}

Fonte: dados da pesquisa (2013) 
Com base na análise da Tabela 2, verifica-se que o indicador de desempenho foi a técnica de diagnóstico mais utilizada entre as avaliações analisadas. Ela esteve presente $80,82 \%$ das avaliações, seguido da técnica SWOT, com $27,39 \%$. As técnicas de análise de processos e análise stakeholder também foram bastante utilizadas nas avaliações. Além disso, verificou-se que a média de uso das técnicas por avaliação é de 2,20 e que 11 técnicas de diagnóstico foram utilizadas acima da média e 3 foram utilizadas abaixo, variando de uma a duas, são elas Mapa Cognitivo e RECl, com frequência de uso (01) e Matriz de Riscos (02).

Os resultados observados podem ser justificados pelo fato da técnica de indicador de desempenho ser, dentre as técnicas de diagnóstico analisadas, uma das mais tradicionais, em virtude de sua eficácia e da facilidade de aplicação nos processos de avaliação dos programas.

\subsection{Estratégias metodológicas dos programas governamentais}

A pesquisa permitiu verificar a existência de 6 tipos diferentes de estratégias metodológicas utilizadas nas avaliações dos programas analisados, as quais são apresentadas na Tabela 3.

Tabela 3: Estratégias metodológicas utilizadas nas avaliações dos programas

\begin{tabular}{c|c|c}
\hline $\begin{array}{c}\text { Estratégias } \\
\text { Metodológicas }\end{array}$ & $\begin{array}{c}\text { Quantidade de } \\
\text { avaliações }\end{array}$ & Percentual (\%) \\
\hline Análise Bibliográfica & 73 & 100,00 \\
Análise Normativo & 73 & 100,00 \\
Estudo de Caso & 23 & 31,50 \\
Pesquisa Quase & 01 & 1,37 \\
Experimental & & 13,70 \\
Teste Piloto & 10 & 49,31 \\
Visitas de Estudo ou & 36 & \\
Visitas Exploratória & &
\end{tabular}

Fonte: dados da pesquisa (2013)

A partir da análise da Tabela 3, identifica-se que a análise bibliográfica e a análise normativa foram utilizadas em $100 \%$ das avaliações dos programas governamentais analisados. Esses resultados possibilitam inferir que a análise normativa reflete o comprometimento do órgão de controle com a legislação normativa e expressa um reflexo da auditoria de conformidade. Esse tipo de auditoria apresenta a legislação como critério, já a bibliográfica reflete o esforço da Seprog em inserir um aspecto com base científica.

\subsection{Coleta de dados dos programas governamentais}

A Tabela 4 apresenta os tipos de coleta de dados utilizadas nas avaliações de programas analisadas: 
Tabela 4: Coleta de dados utilizadas nas avaliações de programas

\begin{tabular}{c|c|c}
\hline Tipos de coleta & $\begin{array}{c}\text { Quantidade } \\
\text { de avaliações }\end{array}$ & $\begin{array}{c}\text { Percentual } \\
(\%)\end{array}$ \\
\hline $\begin{array}{c}\text { Entrevistas estruturada, } \\
\text { semi-estruturada e não- }\end{array}$ & 55 & 75,34 \\
estruturada & & \\
Grupo Focal & 06 & 8,22 \\
Painel de Referência & 73 & 100,00 \\
Pesquisa Postal, web- & 47 & 64,38 \\
mail e eletrônica & & \\
\hline
\end{tabular}

Fonte: dados da pesquisa (2013)

Com base na análise realizada da Tabela 4, pode-se constatar que as equipes que desenvolveram as avaliações de programas governamentais analisados, utilizaram o painel de referência em todas as avaliações. Em contrapartida, verifica-se que o grupo focal foi a ferramenta de coleta de dados menos utilizada, a qual foi utilizada em 6 avaliações da amostra pesquisada.

A partir dos resultados observados, verifica-se que essas escolhas de coletas de dados foram realizadas com a finalidade de validar os papeis de trabalho elaborados e os achados encontrados nas auditorias. Essa constatação demonstra zelo dos auditores diante da execução do seu trabalho, possibilitando que as avaliações apresentem aspectos científicos e ganhem maior credibilidade.

\subsection{Análise de dados dos programas governamentais}

A Tabela 5 é responsável por apresentar os tipos de análises de dados utilizados nas avaliações de programas governamentais analisados

Tabela 5: Análise de dados usadas nas avaliações de programas

\begin{tabular}{c|c|c}
\hline Tipo de análise de dados & $\begin{array}{c}\text { Quantidade } \\
\text { de avaliações }\end{array}$ & Percentual \\
\hline $\begin{array}{c}\text { Análise de Conteúdo } \\
\text { Análise de Conglomerados }\end{array}$ & 01 & 1,37 \\
(cluster analysis) & 01 & 1,37 \\
Análise de Frequência & 47 & 64,38 \\
Análise Envoltória de Dados & 01 & 1,37 \\
(DEA) & 55 & 75,34 \\
Análise Qualitativa & 02 & 2,73 \\
Cruzamento de Bases de & & \\
Dados & &
\end{tabular}

Fonte: dados da pesquisa (2013)

A partir da análise da Tabela 5, identifica-se que o TCU tem optado por realizar a análise qualitativa em número maior de avaliações dos programas governamentais. Verificou-se que os 
outros tipos de análises de dados foram utilizados, esporadicamente, em uma ou duas avaliações analisadas.

Esses resultados possibilitam discutir que os auditores têm priorizado análises sem a utilização de ferramentas estatísticas, fato que pode ser justificado por tais profissionais serem mais conservadores na utilização das ferramentas de execução de seu trabalho, tendo em vista que essas técnicas são recentes no campo da auditoria.

\subsection{Equipes responsáveis pela avaliação dos programas}

O estudo realizado permitiu verificar que as 73 avaliações de programas governamentais analisados foram executadas por 490 auditores, o que representa em média 6,71 auditor por avaliação. Com relação ao gênero, identificou-se que 319 auditores são do sexo masculino, o que representa $65,10 \%$ e, 171 do sexo feminino, equivalente a $34,90 \%$.

As equipes de auditoria responsáveis pelas avaliações de programas analisadas foram formadas utilizando o mínimo de 2 auditores (Programas Levantamento da Função Avaliação, da área de Administração Pública e Farmácia Básica, da área de Saúde), e, no máximo 30 auditores, o que ocorreu na avaliação da Ação Alfabetização Solidária, da área da Educação. Verifica-se que o TCU mantém pelo menos 2 auditores na realização de suas auditorias, o que fato que segue os padrões de auditoria internacional (GAO, INTOSAI) e, proporciona maior confiabilidade as auditorias realizadas. Essa situação visa reduzir os conflitos de interesses que podem ser gerados durante o processo de execução das auditorias.

Constatou-se que dos 490 auditores responsáveis pelas avalições dos programas governamentais analisados, 12 pertencem a outras entidades: 5 são do Tribunal de Contas da Bahia, 5 do Tribunal de Contas de Pernambuco, 1 do Tribunal de Contas do Ceará e 1 da AGE/MG. Esses profissionais participaram de 10 avaliações, sendo 5 no ano de 2005 e 5 em 2006. Acrescenta-se a essas informações, que nos exercícios de 2002 a 2004 e 2007 a 2012, o TCU manteve-se fechado ao intercâmbio com outros entes governamentais quanto a compartilhar as suas avaliações de programas não contribuiu. Essa situação não contribuiu para o avanço da disseminação das auditorias operacionais no Brasil.

Além disso, observou-se que $2,04 \%$ dos componentes das equipes são membros externos. Vale ressaltar, que a avaliação do Projeto de Proteção Ambiental e Desenvolvimento Sustentável do Sistema Aquífero Guarani, envolveu os governos da Argentina, Paraguai e Uruguai. Essa situação evidencia que o TCU tem procurado disseminar as práticas de avaliação de programas com outros órgãos, mesmo de forma tímida.

\subsection{Homem Dia Fiscalizações - HDF}

O TCU utiliza a variável HDF para medir a relação de tempo por auditor nas equipes de auditoria. A Tabela 6 apresenta a quantidade de HDF para cada avaliação no período analisado: 
Tabela 6: Quantidade de HDF e de avaliações por exercício - Seprog (2002-2012)

\begin{tabular}{c|c|c|c}
\hline Exercício & $\begin{array}{c}\text { Quantidade de } \\
\text { avaliações }\end{array}$ & HDF & $\begin{array}{c}\text { Média de HDF por } \\
\text { Avaliação Programa }\end{array}$ \\
\hline 2002 & 02 & 188 & 94,00 \\
2003 & 05 & 421 & 84,20 \\
2004 & 11 & 695 & 63,18 \\
2005 & 06 & 408 & 68,00 \\
2006 & 09 & 520 & 57,77 \\
2007 & 02 & 350 & 175,00 \\
2008 & 06 & 1380 & 230,00 \\
2009 & 07 & 975 & 139,28 \\
2010 & 11 & 1628 & 148,00 \\
2011 & 07 & 1146 & 16371 \\
2012 & 07 & 891 & 127,28 \\
\hline
\end{tabular}

Fonte: dados da pesquisa (2013)

A partir da análise da Tabela 6, verifica-se que os HDFs das 73 avaliações de programas analisados variaram no intervalo de 188 (2002) a 1.628 (2010). Além disso, registram um total de 8.602 HDFs e de média 117,83 HDF por avaliação de programa. De modo geral, o TCU tem adotado como padrão a auditoria tradicional (conformidade, legalidade e ou regularidade) que conta com a utilização de uma equipe formada por 2 auditores, prazo de 15 dias úteis, sendo 5 para planejamento, 5 para execução e 5 para relatório, o que resulta em torno de 30 HDFs.

As avaliações de programas não adotam padrões fixos de tempo nem de quantidade de membros por equipe. Quando é comparada à média da HDF de uma avaliação de programas com a de uma auditoria de conformidade, verifica-se que a avaliação de programas consome 392,76\% a mais de HDF. Essas características fazem com que a avaliação de programas seja de longa duração e com alto um custo para o TCU.

\subsection{Solicitações do Congresso Nacional (CN)}

Identificou-se que o Congresso Nacional solicitou ao TCU a realização de 3 avaliações de programas no período analisado, sendo uma em 2008 e duas em 2009, o que evidencia uma baixa demanda, por parte dos congressistas, seja pelo desconhecimento deste tipo de auditoria ou pela tímida atuação do TCU junto aos parlamentares.

Esses resultados contrariam uma tendência mundial (SCOTT, 1995; HOOD, 1996; WALSH, 1996; TRODDEN, 1996), uma vez que em outros países existe grande interesse do parlamento pelas avaliações de programas governamentais, inclusive, o GAO prioriza este tipo de auditoria em relação à auditoria tradicional (RIST, 1989).

\subsection{Deliberações das avaliações dos programas}


Quando são analisadas as deliberações emitidas pelo TCU nas 73 avaliações de programas, verifica-se que o TCU emitiu 1.373 deliberações de melhoria. Esse resultado representa a média de 18,80 deliberação por avaliação. Além disso, o estudo permitiu observar um número de recomendações abaixo da média em 40 avaliações. As recomendações por avaliação variaram de 0, (observada em 5 programas) a 45 (Programa Sistema Único de Segurança Pública, da área de Cidadania). Identificou-se que dos 5 programas que em que não houve deliberações, 4 deles, são levantamentos que direcionaram o TCU para a realização de outras fiscalizações.

Tabela 7: Quantidade de deliberações por área (2002-2012)

\begin{tabular}{c|c|c|c}
\hline Área & $\begin{array}{c}\text { Quantidade } \\
\text { de } \\
\text { deliberações }\end{array}$ & $\begin{array}{c}\text { Avaliação } \\
\text { por área }\end{array}$ & $\begin{array}{c}\text { Média de } \\
\text { deliberações por } \\
\text { área }\end{array}$ \\
\hline Administração Pública & 00 & 01 & 00 \\
Agricultura & 90 & 04 & 22,5 \\
Assistência Social & 246 & 10 & 24,6 \\
Cidadania & 207 & 07 & 29,57 \\
Ciência e Tecnologia & 02 & 02 & 01 \\
Comunicação & 25 & 01 & 25 \\
Direitos Humanos & 16 & 01 & 16 \\
Educação & 62 & 03 & 20,67 \\
Energia Elétrica & 21 & 01 & 21 \\
Esportes & 53 & 03 & 17,66 \\
Gestão Ambiental & 49 & 03 & 16,33 \\
Habitação, Saneamento e & 32 & 03 & 10,67 \\
Urbanismo & & & \\
Indústria, Com. e Serv. & 33 & 03 & 11 \\
Organização Agrária & 41 & 01 & 41 \\
Previdência Social & 17 & 01 & 17 \\
Relações Exteriores & 11 & 02 & 5,5 \\
Saneamento & 41 & 02 & 20,5 \\
Saúde & 303 & 17 & 17,82 \\
Segurança & 47 & 02 & 23,5 \\
Trabalho & 30 & 02 & 15 \\
Transporte & 47 & 04 & 11,75 \\
\hline Fonte: dadosdapesqusa & & &
\end{tabular}

Fonte: dados da pesquisa (2013)

A Tabela 7 apresenta a quantidade de deliberações por área de atuação do TCU e registra uma média de deliberações de 5,04. Constatou-se que 4 avaliações ficaram com deliberações abaixo da média, o que foi interpretado como ótimo desempenho dos programas, tendo em vista que não foi necessário intervenção do TCU. Verificou-se que apesar da área da Saúde possuir o maior número de deliberações (303), seguida das áreas de Assistência Social (246) e Cidadania (207), as áreas que o TCU mais colaborou foram as que apresentam as maiores médias de deliberações, as quais foram: Organização Agrária (41), Cidadania $(29,57)$ e Assistência Social $(24,6)$. 


\subsection{Monitoramento das deliberações}

O TCU realizou 73 monitoramentos nas 73 avaliações realizadas pela Seprog, o que representa uma média de 1 Monitoramento/Avaliação (Memória: $73 / 73=1$ ). No entanto, os monitoramentos realizados ocorreram conforme a Tabela 8.

Tabela 8: Monitoramentos realizados nas avaliações de 2002-2012 e suas implementações

\begin{tabular}{c|c|c|c|c|c|c|c|c}
\hline Monitoramento & Avaliação & Deliberação & \multicolumn{5}{|c}{ Implementadas } \\
& & & Sim & $\%$ & Não & $\%$ & $\begin{array}{c}\text { Não } \\
\text { Sabe }\end{array}$ & $\%$ \\
\hline Não Monitorados & 32 & 371 & - & - & - & - & 371 & 100 \\
$\begin{array}{c}\text { 10 Monitoramento } \\
\text { Até o 20 }\end{array}$ & 17 & 348 & 107 & 24 & 241 & 43 & - & - \\
$\begin{array}{c}\text { Monitoramento } \\
\text { Até o 3o }\end{array}$ & 08 & 447 & 218 & 50 & 229 & 40 & - & - \\
$\begin{array}{c}\text { Monitoramento } \\
\text { Total }\end{array}$ & 73 & 1.373 & 435 & 32 & 567 & 41 & 371 & 27 \\
\hline
\end{tabular}

Fonte: dados da pesquisa (2013)

Com base na análise da Tabela 8, identifica-se que foram emitidas 1.373 deliberações nas 73 avaliações pesquisadas. Dessas, 435 foram implementadas, conforme foi comprovado através de monitoramentos. Acrescenta-se a essas informações, que quando são analisadas as 73 avaliações, 32 não foram monitoradas. Essa situação impacta negativamente no processo de auditoria, uma vez que não foi verificada a implementação de 371 deliberações, o que representa $27 \%$ das deliberações emitidas pelo TCU.

Por mim, verificou-se que 8 avaliações completaram o ciclo proposto (3 monitoramentos). Quando analisamos esse resultado em confronto com as 73 avaliações analisadas, identifica-se que $10,95 \%$ das avaliações passaram pelo ciclo completo de monitoramento. Esse resultado demonstra baixa atuação do TCU no sentido de realizar o ciclo completo de monitoramento. Vale ressaltar, que segundo o manual de auditoria operacional (Brasil, 2010), o início do monitoramento não deve demorar para ser implantado, com a perspectiva de que não seja perdida a expectativa de controle. Por outro lado, não é recomendável que o monitoramento seja estendido por muito tempo.

\section{CONSIDERAÇÕES FINAIS}

A pesquisa em atenção ao objetivo formulado identificou a atuação do TCU na avaliação de programas governamentais em funções do governo no período 2000-2012.

Constatou-se que o TCU contribuiu com o aperfeiçoamento das auditorias operacionais realizadas no período analisado. Essa atuação caracteriza-se como freios e contrapesos na resolução dos conflitos entre gestores públicos e os direitos do cidadão, favorecendo a busca pela excelência na qualidade dos bens e serviços, agregando valor aos recursos investidos, 
auxiliando na tomada de decisão, reduzindo os riscos e promovendo o desenvolvimento nacional.

Verificou-se que 32\% das deliberações do TCU já foram implementadas, situação que evidencia a efetiva contribuição do TCU na evolução dos 73 programas avaliados. Esses resultados são positivos e permitem observar que a presença do controle externo na avaliação dos programas governamentais tem cumprido um papel significativo. Essa atuação do TCU impõe respeito, alerta e inibe ações contrárias à boa prática de gestão dos stakeholders envolvidos no processo de políticas públicas.

O estudo revelou que o TCU utiliza estratégicas metodológicas em suas avaliações, dando ênfase as análises normativa e bibliográfica, realizando a coleta dos dados por meio de entrevistas, pesquisa postal e grupo focal, utilizando o painel de referência com especialistas para validar as fases finais de planejamento e, de execução, para os papeis de trabalho e os achados. Identificou-se que analisa os dados através de análises de frequência e qualitativa; e adiciona ao desenvolvimento das avaliações técnicas de diagnóstico de auditorias, o que confere às avaliações um caráter científico em sintonia com os padrões internacionais.

A partir da pesquisa, verificou-se que as avaliações dos programas governamentais analisados não adotam padrões fixos de tempo em relação à formação da equipe responsáveis pelas auditorias. Os resultados demonstraram que foram consumidos em média 117,83 HDFs, 0 que torna onerosa e de longa duração esse tipo de auditoria para o TCU. Essa situação coloca auditorias com esse enfoque em desvantagem quando comparada com as auditorias tradicionais, que consomem em uma auditoria padrão cerca de $30 \mathrm{HDFs}$.

Por meio da Portaria TCU 253, de 21/12/2012, a Seprog foi transformada em Secretaria de Métodos Aplicados e Suporte à Auditoria - Seaud, modificando o seu foco de secretaria especializada em programa, para uma secretaria repassadora de metodologias que age de forma tutorial. Essa mudança alinha-se à nova forma de atuação do TCU, que adotou a auditoria de governança pública como eixo principal para nova gestão.

Conclui-se que as avaliações de programas do TCU estão em sintonia com a tendência mundial contribuindo com a Administração Pública Federal Brasileira tendo ofertado 1.373 sugestões, cujo impacto nos programas públicos foi advindo da implementação de 435 destas recomendações. Contudo, a despeito da avaliação positiva quanto aos benefícios gerados pela atuação do TCU, a pesquisa identificou que a demanda por este tipo de auditoria, por parte dos congressistas, não está alinhada ao comportamento mundial (NAO, GAO, Corte de Contas da Alemanha e da França).

Por fim, recomenda-se para futuras pesquisas que sejam evidenciadas as ações implementadas a partir das recomendações propostas nas avaliações dos programas governamentais e a evolução dos programas governamentais a partir dessas recomendações. Além disso, sugere-se, ainda, verificar como o TCU passou a realizar suas avaliações de programas de governo após a extinção de sua secretaria especializada - Seprog. 


\section{REFERÊNCIAS}

Aguilar, M. J., \& Ander-Egg, E. (1994). Avaliação de Serviços e Programas Sociais. Petrópolis: Vozes.

Barros, C. C. (2014). O impacto da auditoria de governança na Administração Pública Federal Brasileira: o caso do Acórdão/TCU 1.603/2008. Dissertação de mestrado, Programa Multiinstitucional e Inter-Regional de Pós-Graduação em Ciências Contábeis (UnB/UFRN/UFPB), Universidade Federal do Rio Grande do Norte, Natal, RN, Brasil.

Barzelay, M. (1992). Breaking through bureaucracy: A New Vision for Managing in Government. University of California Press.

(1997). Central Audit Institutions and Performance Auditing: A comparative Analysis of Organizational Strategies in the OECD. Governance, 10(3), 75-87.

Batista, E. M., Barros, C. C., Barros, F. M.C., Melo, O. A. \& Souza, F. J. V. (2014). As Avaliações de Programas Públicos Realizadas Pelo TCU na Área da Saúde. Anais do Congresso Controladoria e Contabilidade USP, São Paulo, SP, Brasil, 14.

Brasil. Constituição (1988): Texto Constitucional de 5 de outubro de1988 com alterações adotadas pelas emendas constitucionais 1/92 a 68/2011. Ed. Atual. Brasília: Senado Federal.

TCU Histórico da ANOp. Recuperado de http://portal2.tcu.gov.br/portal/page/portal/TCU/comunidades/programas_governo

Calmon, K. M. N.. A. (1997). Avaliação de programas como instrumento de aprendizagem organizacional: o caso do PROSEGE. Dissertação de Mestrado, Universidade de Brasília, Brasília, DF, Brasil.

Hedler, H. C. (2007). Meta-avaliação em de Auditorias de Natureza Operacional do Tribunal de Contas da União: Um estudo sobre auditorias de Programas Sociais. Tese de doutorado, Universidade de Brasília, Brasília, DF, Brasil.

Hood, C. (1999). Regulation inside government. Waste-Watchers, Quality Police and SleazeBusters. New York: Oxford University Press.

Matias Pereira, J. (2010). Governança no setor público. São Paulo: Atlas.

Moore, M. (1995). Creating public value: management in government. Cambridge: Havard University Press.

- (2007). Criando valor público por meio de parcerias público-privadas. Revista do Serviço Público Brasília 58 (2), 151-179.

Pollit, C.( 1999). Performance or compliance? Performance Audit and Public management in Five Countries. Oxford: Oxford University Press.

Rezende, F., Cunha, A. \& Cardoso, R. L. (2010). Custos no setor público. Revista de Administração Pública, Rio de Janeiro, 44 (4), 789-790.

Rist, R. C. (1989). Management acountability: the signals sent by auditing and evaluation. Journal of Public Policy, 9 (3), 355-369.

Scott, W. R. (1995). Institutions and Organizations. Thousand Oaks, CA: Sage.

Sedgwick, S. (1993). When Does an Audit Become an Evaluation? Canberra Bulletin of Public Administration, September, 148-151.

Vieira, A. M.\& Gomes, M. B. (2002). Instituições centrais de auditoria e auditoria de desempenho: uma análise comparativa das estratégias organizacionais na OCDE. Revista do TCU. Brasília, 7-24.

Weiss, C.( 1998). Evaluation. Methods for studying Programs and Policies. Upper Sadle River, Prentice-Hall. 